\title{
The Experimental Teaching Based on Hybrid Cloud Management System Research
}

\author{
Donglin Chen \\ Institute of e-Business, Wuhan University of \\ Technology \\ Wuhan, China \\ chendl@whut.edu.cn
}

\author{
Ling Chen \\ Institute of e-Business, Wuhan University of \\ Technology \\ Wuhan, China \\ cchenlling9005@163.com
}

\author{
Qiaofen Ji \\ Institute of e-Business, Wuhan University of Technology \\ Wuhan, China \\ jiqiaofen@126.com
}

\begin{abstract}
Cloud computing cost-effective, and scalable characteristics can be an effective solution to teaching college laboratory teaching uneven distribution of resources, updates slow, the shared low degree. Analysis of cloud computing at home and abroad in the application on the basis of education, the paper put forward and design college laboratory teaching management system based on hybrid cloud management function, and provide a good guide for colleges and universities to achieve cloud computing experimental teaching management.
\end{abstract}

Keywords-hybrid cloud; experimental teaching management system; business support system; operations support system

\section{I .Introduction}

The experimental teaching which takes an indispensable part in colleges and universities teaching process plays an important role in training students' practical ability and creative spirit. However, nowadays a lot of domestic university experimental teaching links exist many problems, such as the experimental teaching resources uneven distribution, update slowly, utilization rate is low, the lack of resources sharing, the mode of management is backward and so on, which makes the experiment teaching become weak link, and is unfavorable to the cultivation of talents. Therefore, how to efficient utilization, fully integrate the existing experimental teaching resources, and reduce the cost of teaching, improve teaching quality, are the present domestic universities' challenges [1].

In 2008 at the China education technology society's annual meeting, professor Li in Shanghai Normal University first put forward the concept of "Cloud Computing Assisted Instruction" and "Clouds Computing Based Education", the cloud computing technology was introduced into the education field, use the "cloud service" auxiliary education teaching [2]. The combination of cloud computing and education becomes the inevitable development trend of future education.

\section{II .Related work}

Experimental teaching model based on cloud computing breakthrough the traditional experimental teaching status subject to the restrictions of time and space. Graham Elementary School in North Carolina, USA and Simtone introduced "universal cloud computing services" to bring the virtual computer desktop to the school. North Carolina State University cooperate with IBM launched the "Virtual Computing Lab (VCL)" cloud computing platforms to provide online educational materials, application software, computing and storage services to colleges, primary and secondary schools [3]. There is a free platform of teaching and research in Seattle, the resources in the cloud platform is set up as the form of "donation", and the cloud platform is composed by the existing resources available in school laboratories, we can achieve access to resources by the portable client [4].

Cloud computing is not only saved the money but also broke the experimental conditions and environmental barriers. Let more of college scientific research personnel, including students to join to the complex experiment and research [5].

\section{III .Based on hybrid cloud experimental teaching management system}

In order to ensure the normal running of hybrid cloud laboratory, we need to do decentralized management of hybrid cloud laboratory resources, applications, user information and so on, therefore, we build the based on hybrid cloud university experimental teaching management system, as it's shown in figure 1: 


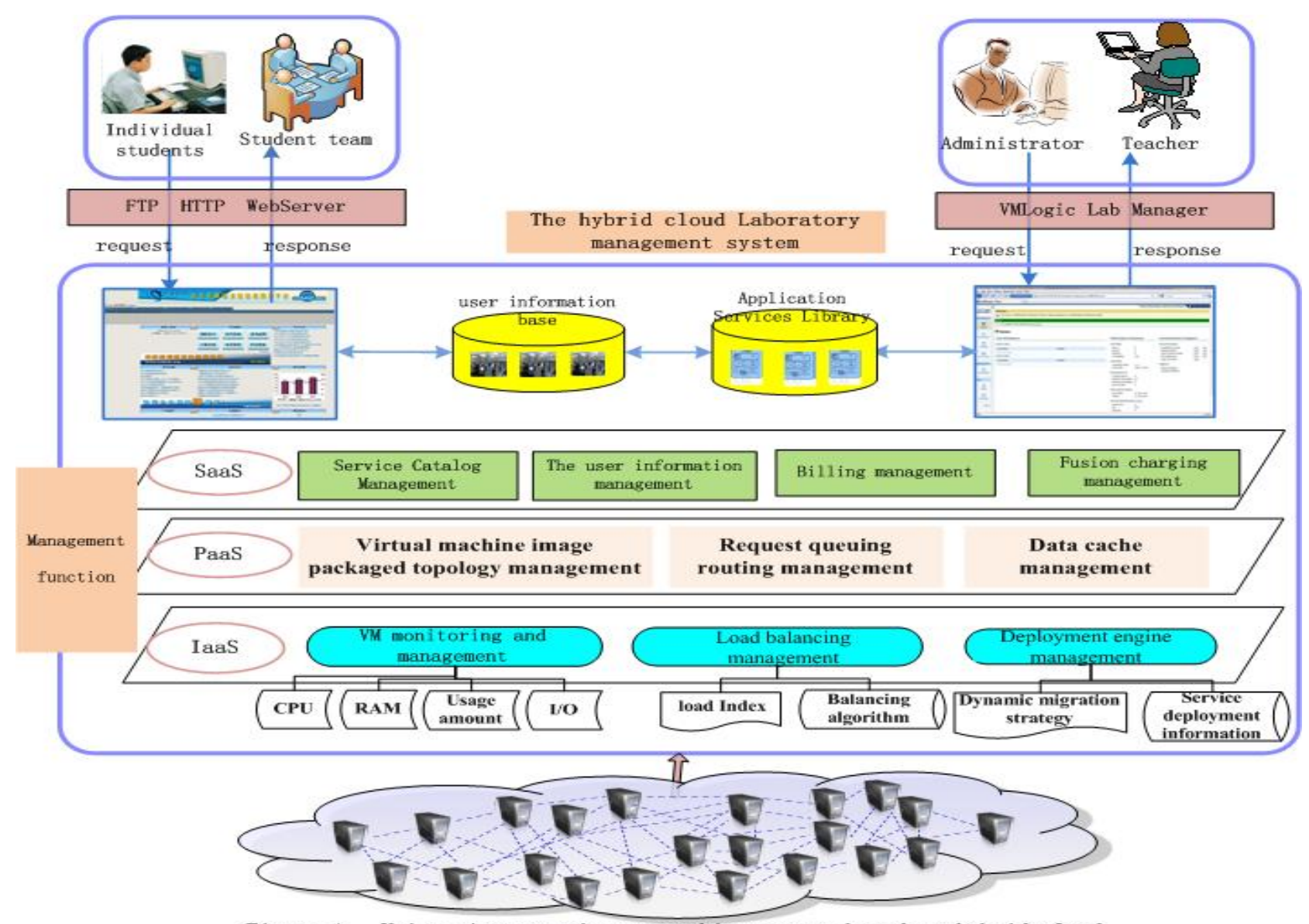

Figure 1 University experiment teaching system based on hybrid cloud

Students can through accessing an FTP server or Web server to enter into the experimental environment, and applying for experiments required resources, waiting for the teacher and administrator audit. The teachers and administrators can deploy the experimental environment by VM Logic Lab Manager, allocate the approval request experimental resources, and view the students in the experimental progress, task execution status. Students only need a browser to enter into the experimental environment, using of hybrid cloud deployment IaaS, PaaS, SaaS services which can be paid according to the actual usage. In order to protect the high reliability of cloud services, cloud service providers (including public cloud providers and private colleges and universities cloud providers) need to carry out a series of management activities. IaaS layer include virtual machine resource status monitoring, the running state of the virtual machine monitor, use metering, load balancing, resource scheduling and management, etc [6]. In the PaaS mainly include virtual machine images packaged topology management, request queuing routing management, data cache management, data backup management, etc. SaaS layer include quality of service (QoS) monitoring, Service Level Association (SLA) proposed monitoring, billing management, etc [7]. In addition, the hybrid cloud laboratory system is in need of the services of directory management, rights management and billing management, and these management functions need the integration of private cloud Academic System, financial system from college and customer relationship management systems from the public cloud to ensure the normal operation of the cloud computing services. Experimental teaching management system includes a service portal, service certification, Business Support System (BSS), Operations Support System (OSS) these four parts, Business Support System include order management, customer management, account management, billing management, supplier management, service catalog management and so on, Operations Support System include resource management, resource allocation, monitoring and management, security management, report management, backup management functions, etc. Research system as shown in figure 2: 


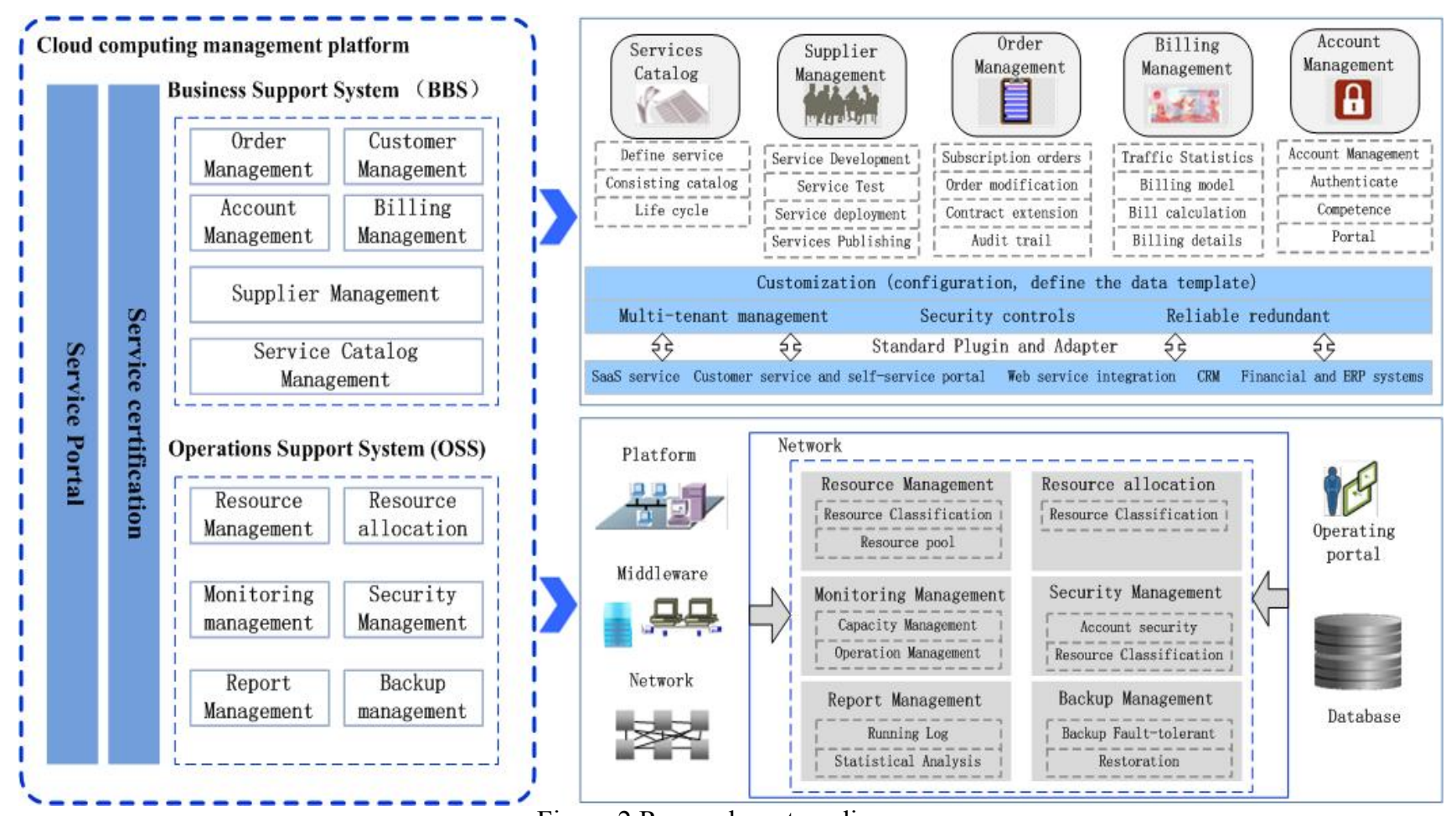

Figure 2 Research system diagram

(1) Business Support System (BSS)

Business Support System includes order management, customer management, account management, billing management, supplier management, service catalog management these functions. The service catalog management can define different services, or a combination of multiple services (such as an integrated application support environment), to compose service catalog and provide different services lifecycle management strategies. Supplier Management allows third-party to do service development, service testing, service deployment on cloud platform and release to the appropriate directory when operators through the audit. Order management includes certification orders, order modification, renewal, and audit trail. Users can query all orders, and tracking the usage and consumption record of every order. Accounting management sets pricing rules for different service, the statistical of user traffic, costs are calculated based on the traffic and users use the service price, detail accounting is in accordance with the rules, users can view their billing details. Account management system includes user authentication, permissions set and portal landing, system accounts includes the account of the end-user accounts and third-party suppliers. User management includes multi-tenant (organization) design, providing the definition of organizations.

(2) Operations Support System (OSS)

Operations support System including resource management, resource allocation, monitoring and management, security management, report management, backup management functions. Resource management composes the infrastructure of the same resource group to a virtual pool of resources which is based on different resource attributes (such as geographical, SLA indicators) defined resource group, and the resource pool will be real-time monitored. Resource allocation is in accordance with different loads and scheduling policies, and distributes different services (load) to different resource pools. Monitoring management provides resource capacity monitoring and service performance, health status monitoring of the overall platform. The safety management cloud computing platform provides the perfect means to ensure the security and isolation of each project and. Users access to the system by two channels: to access the Web management interface and access to project virtual machine. It needs the user name and password to access to the Web interface. By the following means to control accessing to the virtual machine: the external interface of the system by VPN devices to authenticate the user. The isolation of the virtual machine is guaranteed by the virtualization engine itself. Report Manager generates a system running log, while providing the resources statistics, revenue statistics, trend analysis, etc.

\section{IV .Conclusion}

Establish the university experimental teaching management system based on hybrid cloud, realized the university experimental teaching platform construction and sharing of resources. It not only saved the money and broke through the traditional experiment teaching time and space limit, but also broke the experimental conditions and environmental barriers, this mode of education field 
especially practice teaching has the profound influence. Universities should give full play to take advantages of cloud computing in education field, and make great efforts to explore more cloud computing education application, further in wider range to improve the experimental teaching effect.

\section{Acknowledgment}

This research was supported by the National Natural Science Fund of China under Grant71172043, 71072077; the National Technology R\&D Program 2011BAH16B02; the Fundamental Research Funds for the Central Universities and Teaching and research projects of WHUT.

\section{Reference}

[1] Yang Qian,Jun Dai,Xiaoyan Liao. Oriented to information resource management of cloud computing performance analysis.Library and information, 2009( 4) : 53- 56.

[2] Jiahou Li.Toward educational technology "cloud" services.Journal of Distance Education, 2008 (03):79.

[3] Chenhui Huang, Yongqin Lin.Research and Implementation based on cloud computing virtual computer lab. Research and Exploration in Laboratory, 2010, 29(11):178-180.

[4] Justin Cappos,Ivan Beschastnikh,Arvind Krishnamurthy,et al.Seattle:A platform for educational cloud computing. Proceedings of the 40th ACM technical symposium, 2009(3):111-115.

[5] Minchao Chen,Lijia Shen. Based on the resources of the collaborative learning instructional design and practice.China Educational Technology, 2010(12):96-98.

[6] Yi Wang,Hong An. Cloud learning age teacher virtual learning community construction. China Educational Technology, 2010(1):118-122.

[7] Jiagui Zhang,Zhexin Cao. Vocational colleges shared professional teaching resource library building reseach. Modern Educational Technology, 2009(7):51-54. 